\title{
Uplifting $\mathrm{AdS}_{3} / \mathrm{CFT}_{2}$ to flat space holography
}

\section{Adam Ball, Elizabeth Himwich, Sruthi A. Narayanan, Sabrina Pasterski and Andrew Strominger}

Center for the Fundamental Laws of Nature, Harvard University, Cambridge, MA 02138, U.S.A.

E-mail: aaball@g.harvard.edu, himwich@g.harvard.edu, sruthi_narayanan@g.harvard.edu, spasterski@fas.harvard.edu, strominger@physics.harvard.edu

Abstract: Four-dimensional (4D) flat Minkowski space admits a foliation by hyperbolic slices. Euclidean $\mathrm{AdS}_{3}$ slices fill the past and future lightcones of the origin, while $\mathrm{dS}_{3}$ slices fill the region outside the lightcone. The resulting link between $4 \mathrm{D}$ asymptotically flat quantum gravity and $\mathrm{AdS}_{3} / \mathrm{CFT}_{2}$ is explored in this paper. The $4 \mathrm{D}$ superrotations in the extended $\mathrm{BMS}_{4}$ group are found to act as the familiar conformal transformations on the 3D hyperbolic slices, mapping each slice to itself. The associated 4D superrotation charge is constructed in the covariant phase space formalism. The soft part gives the $2 \mathrm{D}$ stress tensor, which acts on the celestial sphere at the boundary of the hyperbolic slices, and is shown to be an uplift to $4 \mathrm{D}$ of the familiar 3D holographic $\mathrm{AdS}_{3}$ stress tensor. Finally, we find that $4 \mathrm{D}$ quantum gravity contains an unexpected second, conformally soft, dimension $(2,0)$ mode that is symplectically paired with the celestial stress tensor.

KeYwords: AdS-CFT Correspondence, Space-Time Symmetries

ArXIV EPRINT: 1905.09809 


\section{Contents}

1 Introduction 1

2 Preliminaries 3

3 Superrotation vector fields $\quad 4$

4 Covariant phase space charge $\quad 5$

4.1 Boundary charge 5

4.2 Linearized bulk charge 6

$\begin{array}{lll}4.3 & \text { Exact bulk charge } & 7\end{array}$

$\begin{array}{lll}5 & \text { Massive point particles } & 7\end{array}$

$\begin{array}{lll}6 & \text { Subleading soft theorem } & 9\end{array}$

$\begin{array}{lll}7 & \text { Celestial stress tensor } & 10\end{array}$

8 Dual stress tensor $\quad 11$

A Linearized Einstein equations 12

\section{Introduction}

The metric for flat 4D Minkowski space $\left(M_{4}\right)$ in hyperbolic coordinates is

$$
d s^{2}=-d \tau^{2}+\tau^{2}\left(\frac{d \rho^{2}}{\rho^{2}}+\rho^{2} d z d \bar{z}\right)
$$

where $\tau$ is the Lorentz-invariant distance from the origin and labels the three-dimensional hyperbolic slices in the parenthesis. In order to cover all of $M_{4}$ we take $\tau$ positive in the future lightcone of the origin, negative in the past lightcone and both $\tau$ and $\rho$ imaginary outside the origin; see figure 1. Equation (1.1) represents $M_{4}$ as a kind of non-compact compactification to $\mathrm{AdS}_{3}$. Hyperbolic slicings have been studied for example in [1-4]. ${ }^{1}$

In this paper, we take inspiration from the prescient paper of de Boer and Solodukhin [1]. These authors conjectured that the infinite-dimensional 2D conformal symmetry of $\mathrm{AdS}_{3}$ quantum gravity should uplift to $M_{4}$ quantum gravity, with separate symmetries for the past and the future. Somewhat later, the existence of such conformal symmetries, coined superrotations, was conjectured in [7-10] by relaxing an overly-restrictive

\footnotetext{
${ }^{1}$ See e.g. $[5,6]$ for an alternate approach to $M_{4}$ holography as the flat space limit of $\mathrm{AdS}_{4}$ quantum gravity rather than an uplift of $\mathrm{AdS}_{3}$ quantum gravity.
} 


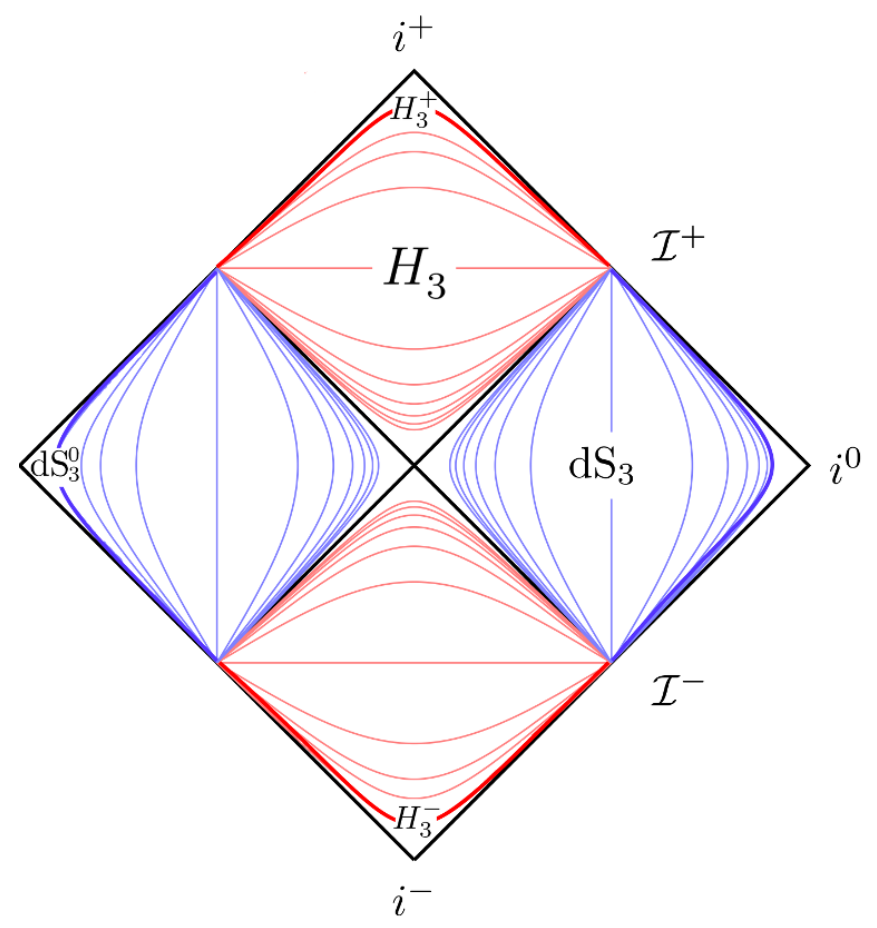

Figure 1. Penrose diagram of hyperbolic slicing of Minkowski space. The slices correspond to surfaces of constant $\tau$. The slices in the past and future lightcones of the origin have the geometry of $H_{3}$, and the slices with spacelike separation from the origin have the geometry of $\mathrm{dS}_{3}$.

assumption about the asymptotic behavior of the gravitational field in the original papers of BMS [11-13]. More recently [14, 15], using the subleading soft theorem of [16], the existence of a single conformal symmetry of quantum gravitational scattering in $M_{4}$ was proved. ${ }^{2}$ The past-future pair of conformal symmetries of $[1,7-10]$ was reduced to a single conformal symmetry by a matching condition required for the consistency of the scattering amplitudes. The reduced symmetry acts in the standard fashion on the celestial sphere at null infinity. This suggests a holographic relation between quantum gravity on $M_{4}$ and an as-yet-to-be-understood "celestial conformal field theory" on the celestial sphere at the boundary.

Despite the natural role played by the hyperbolic slicing (1.1), much of the work on superrotations has used retarded Bondi coordinates (see [3, 4, 17] for important exceptions). The main reason for this is simply that research on asymptotic structure near null infinity over the last half century primarily uses Bondi coordinates and many formulae are readily available; some references are [7-10, 18-20]. However, even the global SL $(2, \mathbb{C})_{\text {Lorentz }}$ subgroup is obscure in these coordinates which are not well-suited for the study of superrotations. A central purpose of this paper is to recast some of the recent results into hyperbolic coordinates and elucidate the connection between $M_{4}$ and $\mathrm{AdS}_{3}$ holography. One hopes that our detailed understanding of AdS holography can be uplifted and applied to flat space holography.

\footnotetext{
${ }^{2}$ We consider only the tree-level subleading soft theorem in this paper.
} 
In section 2 we present formulae and conventions for the hyperbolic foliation of $M_{4}$. In section 3 we show that superrotations have a simple description in terms of vector fields that are tangent to the slices. In section 4 we evaluate the boundary and bulk superrotation charges in the covariant phase space formalism. For the bulk expressions, both the soft parts (which are linear in the metric field) and the hard parts (which involve radiation flux) are evaluated as integrals over hyperbolic slices which hug null infinity where the weak field expansion becomes exact. The soft charges are constructed from uplifts of the holographic stress tensor of $\mathrm{AdS}_{3}$ quantum gravity [21], providing a precise relation between $M_{4}$ and $\mathrm{AdS}_{3}$ holography. In section 5 we explicitly evaluate the hard charge for matter sourced by point particles, and find that it reduces to an integral of the subleading soft factor [16]. Section 6 demonstrates that the total charge conservation, which involves contributions from two $H_{3}$ slices and one $\mathrm{dS}_{3}$ slice, is equivalent to the subleading soft theorem. In section 7 we relate the soft covariant charges to the celestial stress tensor. Section 8 identifies a weight $(2,0)$ mode which is not pure gauge and has a canonical symplectic pairing with the superrotation Goldstone mode. This new $(2,0)$ mode is potentially related to new conformally soft theorems and symmetries, but further investigations are left to future work. The appendix gives details of the linearized Einstein equation in the hyperbolic slicing.

\section{Preliminaries}

In hyperbolic coordinates $(\tau, \rho, z, \bar{z})$ the Minkowski metric takes the form

$$
d s^{2}=-d \tau^{2}+\tau^{2}\left(\frac{d \rho^{2}}{\rho^{2}}+\rho^{2} d z d \bar{z}\right) .
$$

These are related to the usual Cartesian coordinates

$$
d s^{2}=-\left(d X^{0}\right)^{2}+\left(d X^{1}\right)^{2}+\left(d X^{2}\right)^{2}+\left(d X^{3}\right)^{2}
$$

by

$$
\begin{aligned}
& \tau=\sqrt{\left(X^{0}\right)^{2}-\left(X^{1}\right)^{2}-\left(X^{2}\right)^{2}-\left(X^{3}\right)^{2}} \\
& z=\frac{X^{1}+i X^{2}}{X^{0}+X^{3}} \\
& \rho=\frac{X^{0}+X^{3}}{\sqrt{\left(X^{0}\right)^{2}-\left(X^{1}\right)^{2}-\left(X^{2}\right)^{2}-\left(X^{3}\right)^{2}}},
\end{aligned}
$$

with inverse

$$
\begin{aligned}
X^{0} & =\frac{1}{2} \tau \rho\left(1+z \bar{z}+\rho^{-2}\right) \\
X^{1} & =\frac{1}{2} \tau \rho(z+\bar{z}) \\
X^{2} & =-\frac{i}{2} \tau \rho(z-\bar{z}) \\
X^{3} & =\frac{1}{2} \tau \rho\left(1-z \bar{z}-\rho^{-2}\right) .
\end{aligned}
$$


The hyperbolic coordinates represent Minkowski spacetime as a foliation (labelled by $\tau$ ) of $3 \mathrm{D}$ constant curvature hyperbolic spaces. We label the spacelike slices in the future (past) lightcone of the origin by $\tau>0(\tau<0)$. We are especially interested in the $\pm \tau \rightarrow \infty$ slices which approach $\mathcal{I}^{ \pm}$. We denote them by $H_{3}^{ \pm}$. The de Sitter slices at spacelike separations from the origin are labelled by positive imaginary $\tau$. The asymptotic $\tau \rightarrow i \infty$ slice is denoted $\mathrm{dS}_{3}^{0}$. This is illustrated in figure 1 . The $\rho=\infty$ boundary of $H_{3}^{+}$(located at $u=0$ on $\mathcal{I}^{+}$in Bondi coordinates) will be referred to as the "future celestial sphere" and denoted $\mathcal{C S}^{+}$. The analogously defined past celestial sphere will be denoted $\mathcal{C S}^{-}$.

The nonzero connection coefficients are

$$
\begin{aligned}
& \Gamma_{\rho \rho}^{\tau}=\frac{\tau}{\rho^{2}}, \quad \Gamma_{z \bar{z}}^{\tau}=\frac{\rho^{2} \tau}{2}, \quad \Gamma_{\rho \tau}^{\rho}=\frac{1}{\tau}, \quad \Gamma_{\rho \rho}^{\rho}=-\frac{1}{\rho} \\
& \Gamma_{z \bar{z}}^{\rho}=-\frac{\rho^{3}}{2}, \quad \Gamma_{z \tau}^{z}=\frac{1}{\tau}, \quad \Gamma_{z \rho}^{z}=\frac{1}{\rho}, \quad \Gamma_{\bar{z} \tau}^{\bar{z}}=\frac{1}{\tau}, \quad \Gamma_{\bar{z} \rho}^{\bar{z}}=\frac{1}{\rho} .
\end{aligned}
$$

\section{Superrotation vector fields}

3D Euclidean quantum gravity on an asymptotically hyperbolic space $H_{3}$ has a conformal symmetry which acts as $[21,22]$

$$
\zeta_{Y}=Y^{z} \partial_{z}-\frac{1}{2} \partial_{z} Y^{z} \rho \partial_{\rho}-\frac{1}{2 \rho^{2}} \partial_{z}^{2} Y^{z} \partial_{\bar{z}}
$$

where $Y^{z}$ is a conformal Killing vector. This is the conformal symmetry of the holographically dual $\mathrm{CFT}_{2}$ which lives on the $S^{2}$ boundary [23, 24]. This 3D vector field lifts to 4D, where it maps the hyperbolic slices to themselves and generates the superrotations of $4 \mathrm{D}$ quantum gravity in asymptotically flat space $[1,8,14,16]$. In hyperbolic coordinates only one component of the $4 \mathrm{D}$ metric (2.1) is transformed:

$$
\mathcal{L}_{Y} g_{z z}=-\frac{\tau^{2}}{2} \partial_{z}^{3} Y^{z}
$$

This term is independent of $\rho$ and therefore sub-subleading in the large $\rho$ expansion of the metric. In the $3 \mathrm{D}$ case, this component of the metric is proportional to the holographic $2 \mathrm{D}$ stress tensor in the Fefferman-Graham construction [21, 25, 26].

A special role will be played in the following by the choice of vector field

$$
Y^{z}=\frac{1}{w-z}
$$

We define

$$
\zeta_{w} \equiv \zeta_{Y=\frac{1}{w-z}}=\frac{1}{w-z} \partial_{z}-\frac{1}{2(w-z)^{2}} \rho \partial_{\rho}-\frac{1}{\rho^{2}(w-z)^{3}} \partial_{\bar{z}}
$$

Any more general superrotation vector field $\zeta_{Y}$ can then easily be obtained from $\zeta_{w}$ via the relation

$$
\zeta_{Y}(z)=\frac{1}{2 \pi i} \oint d w Y^{w} \zeta_{w}(z) .
$$




\section{Covariant phase space charge}

In this section we compute the covariant phase space charge $\mathcal{Q}^{+}\left(\zeta_{Y}\right)$ as developed in a number of references including [27-34]. Under suitable conditions, the charge $\mathcal{Q}^{+}\left(\zeta_{Y}\right)$ generates (via Dirac brackets or commutators) the superrotations on spacelike surfaces ending at the future celestial sphere $\mathcal{C S}^{+}$. The action of the superrotation charge does not preserve the standard Bondi news falloffs, which means that it does not map the standard phase space (considered in e.g. [35]) to itself. A complete treatment of superrotations will eventually involve an enlarged phase space and associated bracket, which is an interesting and important problem beyond the scope of this paper. Here, we will only use the finite and conserved superrotation charges of states in the standard phase space. For simplicity, we will also restrict to situations in which the Bondi news vanishes on $\mathcal{C S}^{+}$.

\subsection{Boundary charge}

The boundary charge is given by the formula in e.g. [30, 31]

$$
\mathcal{Q}^{+}=-\frac{1}{16 \pi} \int_{\mathcal{C S}^{+}} * F=\lim _{\rho \rightarrow \infty} \frac{1}{32 \pi} \int d^{2} z \rho^{3} \tau F_{\tau \rho},
$$

where

$$
F_{\mu \nu}=\frac{1}{2} \nabla_{\mu} \zeta_{\nu} h+\nabla_{\mu} h_{\nu}{ }^{\lambda} \zeta_{\lambda}+\nabla_{\lambda} \zeta_{\mu} h_{\nu}{ }^{\lambda}+\nabla_{\lambda} h_{\mu}{ }^{\lambda} \zeta_{\nu}+\nabla_{\nu} h \zeta_{\mu}-(\mu \leftrightarrow \nu)
$$

with $\mu, \nu=0,1,2,3$. Here $h_{\mu \nu}$ denotes the linearized, on-shell metric perturbations

$$
g_{\mu \nu}=\eta_{\mu \nu}+h_{\mu \nu}
$$

where $\eta_{\mu \nu}$ is given in (2.1). Before proceeding further, in order to avoid long expressions, we make the radial gauge choice

$$
h_{\tau \mu}=0,
$$

which can also be written $X^{\mu} h_{\mu \nu}=0$. Inserting the expression (3.1) for the superrotation vector field and using radial gauge (4.4) we find

$$
\rho^{3} \tau F_{\tau \rho}=\left(\tau \partial_{\tau}-2\right)\left[\rho^{3} Y^{z} h_{\rho z}-\frac{\rho}{2} \partial_{z}^{2} Y^{z} h_{\rho \bar{z}}+2 \partial_{z} Y^{z} h_{z \bar{z}}\right] .
$$

Under the integral we may integrate by parts with respect to $z$, yielding the expression

$$
\rho^{3} \tau F_{\tau \rho}=Y^{z}\left(\tau \partial_{\tau}-2\right)\left[\rho^{3} h_{\rho z}-\frac{\rho}{2} \partial_{z}^{2} h_{\rho \bar{z}}-2 \partial_{z} h_{z \bar{z}}\right] .
$$

As in [3], the boundary conditions are chosen to ensure that the charge is $\tau$-independent and finite for $\rho \rightarrow \infty$, so that it does not depend on a choice of slice. Finiteness of the charge requires that the leading $\rho$ behavior is $h_{\rho z} \sim \rho^{-3}, h_{z \bar{z}} \sim \rho^{0}$, which is compatible with the

\footnotetext{
${ }^{3} \mathrm{~A}$ time translation can always be used to position the two-sphere $\mathcal{C S}^{+}$at early times before any news has emerged on $\mathcal{I}^{+}$. On the other hand, primaries in a conformal basis [36] typically have divergences in the radiation flux at $\mathcal{C S}^{+}$[37]. Our analysis would require modifications to handle such cases, including additions to the charge as discussed in [32].
} 
linearized analysis in the appendix. Moreover we assume that the Bondi news vanishes at $\mathcal{C S}^{+}$. Otherwise, as mentioned above, there are correction terms to the charge [32]. The finite and $\tau$-independent final boundary expression for the superrotation charge is

$$
\mathcal{Q}^{+}\left(\zeta_{Y}\right)=-\frac{1}{16 \pi} \lim _{\rho \rightarrow \infty} \int_{\mathcal{C S}^{+}} d^{2} z Y^{z}\left[\rho^{3} h_{\rho z}^{(0)}-2 \partial_{z} h_{z \bar{z}}^{(0)}\right],
$$

where the superscript (0) indicates the $\tau$-independent piece of the given metric component.

\subsection{Linearized bulk charge}

Having found an expression for the charge $\mathcal{Q}^{+}$as a surface integral over $\mathcal{C S}^{+}$, we now write a bulk expression for the linearized charge as an integral over $H_{3}^{+}$. This involves integrating by parts and using the linearized vacuum Einstein equations. We denote the linearized charge as $\mathcal{Q}_{S}^{+}(\zeta)$ because, as we shall see, it is the same as the soft part of the full nonlinear charge. The nonlinearities are incorporated in the next subsection, where we also discuss the validity of the linearized approximation.

Starting with the boundary definition of the linearized charge $\mathcal{Q}_{S}^{+}(\zeta)$, the desired bulk expression follows from an application of Stokes's theorem and the linearized constraint equations. By construction the bulk charge is the symplectic product of the metric variation $\mathcal{L}_{\zeta} g_{\mu \nu}$ produced by $\zeta$ with the linearized metric perturbation $h_{\mu \nu}$,

$$
\mathcal{Q}_{S}^{+}(\zeta)=\left(\mathcal{L}_{\zeta} g, h\right)_{H_{3}^{\tau}}=\int_{H_{3}^{\tau}} d \Sigma^{\mu} P^{\nu \lambda \gamma \sigma} \mathcal{L}_{\zeta} g_{\nu \lambda} \overleftrightarrow{\nabla}_{\mu} h_{\gamma \sigma}
$$

where $(,)_{M_{3}}$ is the symplectic product on a three-manifold $M_{3}, H_{3}^{\tau}$ is any hyperbolic slice of given $\tau$ and the required components of $P$ (given in full in [32]) are given below. Since the symplectic product is conserved on-shell (assuming appropriate smoothness conditions at $\mathcal{C S}^{+}$) this expression does not depend on the choice of hyperbolic slice $\tau$. We will take $\tau \rightarrow \infty$. In the quantum theory, $h_{\mu \nu}$ then becomes a free field operator, and commutators with $\mathcal{Q}_{S}^{+}$formally generate linearized superrotations of the metric on $\mathrm{H}_{3}^{+}$.

In the case at hand, the only nonzero component of the metric variation is (3.2) and we need only the component $P^{z z \bar{z} \bar{z}}=\frac{1}{8 \pi \tau^{4} \rho^{4}}$. The linearized charge reduces to the simple expression

$$
\mathcal{Q}_{S}^{+}\left(\zeta_{Y}\right)=\frac{1}{8 \pi} \int_{H_{3}^{+}} \frac{d^{2} z d \rho}{\tau^{2} \rho^{3}} \mathcal{L}_{Y} g_{z z} h_{\bar{z} \bar{z}}^{(0)}=-\frac{1}{16 \pi} \int_{H_{3}^{+}} \frac{d^{2} z d \rho}{\rho^{3}} \partial_{z}^{3} Y^{z} h_{\bar{z} \bar{z}}^{(0)},
$$

where $h_{\bar{z} \bar{z}}^{(0)}$ is the $\tau$-independent part of $h_{\bar{z} \bar{z}}$.

We note that $\mathcal{L}_{Y} g_{z z}$, as given in (3.2), involves only the order $\rho^{0}$ metric perturbation, ${ }^{4}$ which has been identified [21] as the holographic stress tensor in the context of $\mathrm{AdS}_{3}$ quantum gravity. This gives a precise connection of the superrotation generators for $M_{4}$ quantum gravity as an uplift of the generator of conformal transformations for $\mathrm{AdS}_{3}$. More specifically, the soft part of the charge which generates $4 \mathrm{D}$ superrotations in the causal domain of $\mathrm{H}_{3}^{+}$is the symplectic product on the 3D hyperbolic slice of the linearized 4D metric perturbation with the $Y^{z}$-variation of the 3D holographic stress tensor.

\footnotetext{
${ }^{4}$ In section 5 , to facilitate the connection to the soft theorem, a physically equivalent vector field $\zeta_{w}^{\prime}=$ $\zeta_{w}\left(1+\mathcal{O}\left(\frac{1}{\rho^{2}}\right)\right)$ which differs at further subleading orders is introduced.
} 


\subsection{Exact bulk charge}

In the previous subsection, the surface charge $\mathcal{Q}^{+}\left(\zeta_{Y}\right)$ on $\mathcal{C S}^{+}$was reexpressed as a bulk integral over $H_{3}^{+}$in the linearized approximation. For a generic slice in a generic asymptotically flat spacetime ending on $\mathcal{C S}^{+}$, nonlinear corrections are important, and there is no useful bulk expression for the charge. However, it is natural to take $\tau \rightarrow \infty$, in which case (assuming no stable black holes) the slice hugs $\mathcal{I}^{+}$, the fields become weak, and corrections to the linearized approximation are easily incorporated.

In order to obtain the bulk expression on $H_{3}^{+}$from the boundary expression on $\mathcal{C S}^{+}$ one integrates by parts and uses the constraint equations $G_{\tau \mu}=8 \pi T_{\tau \mu}$. In the linearized approximation, ${ }^{5}$ the nonlinear terms on the left hand side and the entire right hand side are set to zero. In the full theory, the constraints reduce (for $\tau \rightarrow \infty$ ) to

$$
-16 \pi T_{\tau \mu}=-2 G_{\tau \mu}=\square h_{\tau \mu}-\nabla_{\tau} \nabla_{\alpha} h_{\mu}{ }^{\alpha}-\nabla^{\alpha} \nabla_{\mu} h_{\tau \alpha}+\nabla_{\tau} \nabla_{\mu} h+\eta_{\tau \mu} \nabla_{\alpha} \nabla_{\beta} h^{\alpha \beta}-\eta_{\tau \mu} \square h,
$$

where the stress tensor is understood to contain both matter contributions and the quadratic gravity wave stress tensor. Corrections which are cubic or higher in $h_{\mu \nu}$ vanish for $\tau \rightarrow \infty$. The full expression for the charge is then

$$
\mathcal{Q}^{+}(\zeta)=\mathcal{Q}_{S}^{+}(\zeta)+\mathcal{Q}_{H}^{+}(\zeta)
$$

where $\mathcal{Q}_{S}^{+}(\zeta)$ is given in (4.8) and the hard charge is

$$
\begin{aligned}
\mathcal{Q}_{H}^{+}(\zeta) & =\int_{H_{3}^{+}} d \Sigma^{\mu} \zeta^{\nu} T_{\mu \nu} \\
& =-\frac{1}{2} \int_{H_{3}^{+}} d^{2} z d \rho \rho \tau^{3} T_{\tau \mu} \zeta^{\mu}
\end{aligned}
$$

For $\zeta_{Y}$ as in (3.1), (4.12) becomes

$$
\begin{aligned}
\mathcal{Q}_{H}^{+}\left(\zeta_{Y}\right) & =-\frac{1}{2} \int_{H_{3}^{+}} d^{2} z d \rho \rho \tau^{3}\left[T_{\tau z} Y^{z}-\frac{\rho}{2} T_{\tau \rho} \partial_{z} Y^{z}-\frac{1}{2 \rho^{2}} T_{\tau \bar{z}} \partial_{z}^{2} Y^{z}\right] \\
& =-\frac{1}{2} \int_{H_{3}^{+}} d^{2} z d \rho \rho \tau^{3} Y^{z}\left[T_{\tau z}+\frac{\rho}{2} \partial_{z} T_{\tau \rho}-\frac{1}{2 \rho^{2}} \partial_{z}^{2} T_{\tau \bar{z}}\right] .
\end{aligned}
$$

Since the matter stress tensor generates diffeomorphisms on the matter fields, this manifestly generates the hard action of the superrotations.

\section{Massive point particles}

In this section we compute the hard charge for a collection of $N$ massive point particles with inertial trajectories, which are given in Cartesian coordinates by

$$
x_{k}^{\mu}(\lambda)=\frac{p_{k}^{\mu}}{m_{k}} \lambda+b_{k}^{\mu},
$$

\footnotetext{
${ }^{5}$ The linearized vacuum equations in hyperbolic coordinates are given in appendix A.
} 
where $k=1, \ldots, N$ and $p_{k}^{2}=-m_{k}^{2}$. We follow the analogous treatment of massless point particles presented in [38]. The massive point particle trajectories asymptote at late times to a fixed point $\left(\rho_{k}, z_{k}, \bar{z}_{k}\right)$ on $H_{3}^{+}$with $\lambda=\tau$. In the coordinates (2.3) this point is determined by

$$
\lim _{\tau \rightarrow \infty} \frac{1}{\tau} x_{k}^{\mu}(\tau)=\frac{p_{k}^{\mu}}{m_{k}}=\frac{\rho_{k}}{2}\left(\begin{array}{c}
1+z_{k} \bar{z}_{k}+\rho_{k}^{-2} \\
z_{k}+\bar{z}_{k} \\
-i\left(z_{k}-\bar{z}_{k}\right) \\
1-z_{k} \bar{z}_{k}-\rho_{k}^{-2}
\end{array}\right) .
$$

The stress tensor of the $k$ th particle is

$$
T_{k}^{\mu \nu}(X)=\int d \lambda \frac{p_{k}^{\mu} p_{k}^{\nu}}{m_{k}} \delta^{(4)}\left(X-x_{k}(\lambda)\right)
$$

Substituting into the first line of (4.12) we find the simple expression

$$
\mathcal{Q}_{H}^{+}(\zeta)=-\left.\lim _{\lambda \rightarrow \infty} \sum_{k}\left(p_{k} \cdot \zeta\right)\right|_{x_{k}^{\mu}(\lambda)} \cdot
$$

To easily connect to the soft theorem, we use the vector field [37, 39]

$$
\zeta_{\mu ; w}^{\prime}=\frac{1}{4} \partial_{w}^{3}\left[X^{\nu}\left(q_{\nu} \partial_{\bar{w}} q_{\mu}-q_{\mu} \partial_{\bar{w}} q_{\nu}\right) \log (-q \cdot X)\right],
$$

where $q$ is the null vector that points towards $w$ on $\mathcal{C S}^{+}$,

$$
q^{\mu}=(1+w \bar{w}, w+\bar{w},-i(w-\bar{w}), 1-w \bar{w}) .
$$

This vector field satisfies

$$
\zeta_{w}^{\prime}=\zeta_{w}\left(1+\mathcal{O}\left(\frac{1}{\rho^{2}}\right)\right)
$$

near $\mathcal{C S}^{+}$and hence gives the same total charge as $\zeta_{w}$. Since $\mathcal{Q}^{ \pm}\left(\zeta_{w}^{\prime}\right)=\mathcal{Q}^{ \pm}\left(\zeta_{w}\right)$, the two vector fields have the same Ward identity and conservation law. ${ }^{6}$ The vector field $\zeta_{w}^{\prime}$ arises naturally in the study of conformal primary wavefunctions [36, 37] as well as in the study of massive matter [3]. The utility of $\zeta_{w}^{\prime}$ over $\zeta_{w}$ in the present context is its simple relation to the momentum space version of the subleading soft factor $[3,16]$. We further define polarization tensors

$\varepsilon_{w w}^{\mu \nu}=\varepsilon_{w}^{\mu} \varepsilon_{w}^{\nu}, \quad \varepsilon_{\bar{w} \bar{w}}^{\mu \nu}=\varepsilon_{\bar{w}}^{\mu} \varepsilon_{\bar{w}}^{\nu}, \quad \varepsilon_{w}^{\mu}(w, \bar{w})=\frac{1}{\sqrt{2}}(\bar{w}, 1,-i,-\bar{w}), \quad \varepsilon_{\bar{w}}^{\mu}(w, \bar{w})=\frac{1}{\sqrt{2}}(w, 1, i,-w)$.

One then finds that (5.4) becomes, after significant algebra,

$$
\mathcal{Q}_{H}^{+}\left(\zeta_{w}^{\prime}\right)=\frac{1}{2} \sum_{k} \int d^{2} z \frac{1}{w-z} \partial_{z}^{3}\left[\frac{p_{k}^{\mu} \varepsilon_{\mu \nu ; \bar{z}} J_{k}^{\nu \alpha} q_{\alpha}}{p_{k} \cdot q}\right],
$$

where the tensors

$$
J_{k}^{\mu \nu}=x_{k}^{\mu} p_{k}^{\nu}-x_{k}^{\nu} p_{k}^{\mu}
$$

are the boost and angular momentum charges of the $k$ th particle. The quantity in square brackets in (5.9) is immediately recognizable as the soft factor in the subleading soft graviton theorem.

\footnotetext{
${ }^{6}$ Their soft and hard parts, however, are not separately equal. We find it curious that, even though their difference is trivial, some computations are easier with $\zeta_{w}^{\prime}$ while others are easier with $\zeta_{w}$.
} 


\section{Subleading soft theorem}

In this section we argue that the Ward identity of our charge implies the subleading soft graviton theorem $[3,16]$. In [14] the classical conservation law associated to superrotations is expressed as a sum of integrals over $\mathcal{I}^{ \pm}$in Bondi coordinates,

$$
Q_{S}(Y)+Q_{H}(Y)=0
$$

with

$$
\begin{aligned}
Q_{S}(Y) & =\frac{1}{16 \pi} \int_{\mathcal{I}^{+}} d^{2} z d u Y^{z} u \partial_{z}^{3} N_{\bar{z}}^{z}-\frac{1}{16 \pi} \int_{\mathcal{I}^{-}} d^{2} z d v Y^{z} v \partial_{z}^{3} N_{\bar{z}}^{z} \\
Q_{H}(Y) & =\int_{\mathcal{I}^{+}} d^{2} z d u r^{2} Y_{\bar{z}}\left(T_{u z}-\frac{1}{2} u \partial_{z} T_{u u}\right)-\int_{\mathcal{I}^{-}} d^{2} z d v r^{2} Y_{\bar{z}}\left(T_{v z}-\frac{1}{2} v \partial_{z} T_{v v}\right),
\end{aligned}
$$

where we take $Y^{\bar{z}}=0, N_{z z}$ is the Bondi news, and we raise and lower sphere indices using the round metric on the unit sphere $S^{2}$. It was shown in [14] that the quantum version of this conservation law is equivalent to the subleading soft graviton theorem [16]. This conservation law can be expressed as the equality of two total charges, one incoming and one outgoing.

In the present paper, in contrast, we have three hard and three soft charges associated to the three slices $H_{3}^{+}, \mathrm{dS}_{3}^{0}$, and $H_{3}^{-}$, depicted in figure 1 . We accordingly decompose

$$
\begin{aligned}
& \mathcal{Q}_{S}\left(\zeta_{w}^{\prime}\right)=\mathcal{Q}_{S}^{+}\left(\zeta_{w}^{\prime}\right)+\mathcal{Q}_{S}^{0}\left(\zeta_{w}^{\prime}\right)+\mathcal{Q}_{S}^{-}\left(\zeta_{w}^{\prime}\right) \\
& \mathcal{Q}_{H}\left(\zeta_{w}^{\prime}\right)=\mathcal{Q}_{H}^{+}\left(\zeta_{w}^{\prime}\right)+\mathcal{Q}_{H}^{0}\left(\zeta_{w}^{\prime}\right)+\mathcal{Q}_{H}^{-}\left(\zeta_{w}^{\prime}\right)
\end{aligned}
$$

Here we show $\mathcal{Q}_{S}\left(\zeta_{w}^{\prime}\right)=Q_{S}\left(\frac{1}{w-z}\right)$ and $\mathcal{Q}_{H}\left(\zeta_{w}^{\prime}\right)=Q_{H}\left(\frac{1}{w-z}\right)$, and therefore that the subleading soft graviton theorem is equivalent to the conservation law on hyperbolic slices

$$
\mathcal{Q}_{S}+\mathcal{Q}_{H}=0
$$

First, we show that

$$
\mathcal{Q}_{H}\left(\zeta_{w}^{\prime}\right)=Q_{H}\left(\frac{1}{w-z}\right)
$$

We can consider the hard charge for massive or massless matter. Massive particles cannot reach the asymptotic $\mathrm{dS}_{3}^{0}$ and therefore contribute only to the $\mathcal{Q}_{H}^{ \pm}$charges. As computed in the previous section, the left hand side is

$$
\frac{1}{2} \int d^{2} z \frac{1}{w-z} \partial_{z}^{3}\left[\sum_{k} \frac{p_{k}^{\mu} \varepsilon_{\mu \nu ; \bar{z} \bar{z}} J_{k}^{\nu \alpha} q_{\alpha}}{p_{k} \cdot q}-\sum_{j} \frac{p_{j}^{\mu} \varepsilon_{\mu \nu ; \bar{z} \bar{z}} J_{j}^{\nu \alpha} q_{\alpha}}{p_{j} \cdot q}\right],
$$

where $p_{k}$ are outgoing and $p_{j}$ are incoming momenta. One finds that the same expression holds when we act with the hard charge on massless particles, with the momenta $p_{k}$ taken to be null. This agrees with $Q_{H}$ in (6.2) (see [14]) and shows that the hard charges are the same. 
Next, we wish to verify agreement between the soft terms evaluated in Bondi and hyperbolic coordinates, i.e.

$$
Q_{S}=\mathcal{Q}_{S}^{+}\left(\zeta_{w}^{\prime}\right)+\mathcal{Q}_{S}^{0}\left(\zeta_{w}^{\prime}\right)+\mathcal{Q}_{S}^{-}\left(\zeta_{w}^{\prime}\right)
$$

In order to do so, we rewrite the first line in the Bondi expression (6.2) as

$$
Q_{S}=\int_{\mathcal{I}^{+}} * J+\int_{\mathcal{I}^{-}} * J
$$

with

$$
J=P^{\nu \lambda \gamma \sigma} \mathcal{L}_{\zeta_{w}^{\prime}} g_{\nu \lambda} \nabla_{\mu} h_{\gamma \sigma} d x^{\mu} .
$$

Note the use here of $\nabla_{\mu}$ rather than $\overleftrightarrow{\nabla}_{\mu}$, which appears in the gravitational symplectic pairing (4.8). Since $\int d u u N_{\bar{z} \bar{z}}$ is the subleading soft graviton insertion, and the Bondi news, up to superrotations, falls off faster than $\frac{1}{u}$ (or $\frac{1}{v}$ ) at the boundaries of $\mathcal{I}[14,40]$, we do not expect new soft contributions from "capping" $\mathcal{I}^{ \pm}$at past and future timelike infinity $i^{ \pm}$. The soft charge (6.8) then becomes

$$
Q_{S}=\int_{H_{3}^{+} \cup \mathrm{dS}_{3}^{0} \cup H_{3}^{-}} * J .
$$

Now that we are integrating over a surface without boundary, we are free to switch from $\nabla_{\mu}$ to $\frac{1}{2} \overleftrightarrow{\nabla}_{\mu}$ because they differ by an exact form. The resulting integrand is the same one defining our soft charges, so we have

$$
Q_{S}=\mathcal{Q}_{S}^{+}\left(\zeta_{w}^{\prime}\right)+\mathcal{Q}_{S}^{0}\left(\zeta_{w}^{\prime}\right)+\mathcal{Q}_{S}^{-}\left(\zeta_{w}^{\prime}\right)
$$

Since it has already been shown that the quantum version of (6.1) is the subleading soft graviton theorem, we have demonstrated the desired equivalence of the quantum matrix elements of $\mathcal{Q}_{S}\left(\zeta_{w}^{\prime}\right)+\mathcal{Q}_{H}\left(\zeta_{w}^{\prime}\right)=0$ to the subleading soft graviton theorem.

\section{Celestial stress tensor}

So far we have not explicitly shown that the action of the charge $\mathcal{Q}_{S}\left(\zeta_{w}^{\prime}\right)$, as suggested by the form of (3.2), corresponds to conformal transformations on the celestial sphere. A fast way to do this is to expand the Bondi news in asymptotic graviton creation and annihilation operators and then use the results of [15]. One finds

$$
i \mathcal{Q}_{S}\left(\zeta_{w}^{\prime}\right)=\mathcal{T}_{w w}^{K M R S}
$$

where $\mathcal{T}_{w w}^{K M R S}$ is the subleading soft graviton mode [15]

$$
\mathcal{T}_{w w}^{K M R S} \equiv \frac{3}{\pi \sqrt{32 \pi G}} \lim _{\omega \rightarrow 0}\left(1+\omega \partial_{\omega}\right) \int \frac{d^{2} z}{(w-z)^{4}}\left(a_{-}(\omega q)-a_{+}^{\dagger}(\omega q)\right),
$$

and $a_{-}$and $a_{+}^{\dagger}$ are asymptotic graviton annihilation and creation operators. As shown in [15], by reverse-engineering the subleading soft theorem of [16], normal-ordered insertions 
of $\mathcal{T}_{w w}^{K M R S}$ in the $4 \mathrm{D} \mathcal{S}$-matrix obey the Ward identities of a $2 \mathrm{D}$ stress tensor, and therefore generate conformal transformations of the celestial sphere. In particular, if we pick a contour $C$ and integrate

$$
\frac{1}{2 \pi i} \oint_{C} d w Y^{w} \mathcal{T}_{w w}^{K M R S}
$$

for an arbitrary $Y^{w}(w)$, the corresponding $\mathcal{S}$-matrix insertions generate conformal transformations on the celestial sphere associated to the holomorphic extension of $Y^{w}$ into the interior of $C$. Thus $i \mathcal{Q}_{S}\left(\zeta_{w}^{\prime}\right)$ is the celestial stress tensor.

\section{Dual stress tensor}

In $\mathrm{U}(1)$ gauge theory, large electric gauge transformations $\delta_{\varepsilon}$ on the celestial sphere are generated by a current $J_{w}$ with left/right conformal dimensions $(1,0)[37,41,42]$. This current can be constructed from the symplectic product of the Goldstone mode wavefunction $\delta_{\varepsilon} A_{\mu}$ with the linearized gauge field operator at null infinity. The Goldstone wavefunction has a symplectic partner which is not pure gauge and leads to a second, symplectically conjugate $(1,0)$ current $S_{w}$ [42]. $S_{w}$ is related to large magnetic gauge transformations [43].

We note briefly here that a similar structure exists for the stress tensor $\mathcal{T}_{w w}^{K M R S}$, which, like $J_{w}$, is constructed from the symplectic product with a $(2,0)$ Goldstone mode wavefunction $\delta_{Y} g_{\mu \nu}$. In the normalization conventions of [37], to which we refer the reader for details, the $(2,0)$ Goldstone mode is ${ }^{7}$

$$
h_{\mu \nu ; w w}^{\text {Goldstone }}=-\frac{1}{6}\left[\nabla_{\mu} \zeta_{\nu ; w}^{\prime}+\nabla_{\nu} \zeta_{\mu ; w}^{\prime}\right] .
$$

This wavefunction has a $(2,0)$ symplectic partner that is not pure gauge. The symplectic partner is the $\Delta=2$ conformal primary wavefunction [37], where for general $\Delta$

$$
h_{\mu \nu ; w w}^{\Delta, \pm}\left(X^{\mu} ; w, \bar{w}\right)=\frac{1}{2} \frac{\left[(-q \cdot X) \partial_{w} q_{\mu}+\left(\partial_{\mu} q \cdot X\right) q_{\mu}\right]\left[(-q \cdot X) \partial_{w} q_{\nu}+\left(\partial_{w} q \cdot X\right) q_{\nu}\right]}{(-q \cdot X \mp i \varepsilon)^{\Delta+2}} .
$$

These solutions are labelled by \pm for ingoing versus outgoing, the complex parameter $w$ for the point where the radiation flux crosses the celestial sphere, and $\Delta$ for the $\operatorname{SL}(2, \mathbb{C})$ conformal weight. In hyperbolic coordinates $(\tau, \rho, z, \bar{z}){ }^{8}$

$$
h_{\mu \nu ; w w}^{\Delta, \pm}=\frac{\tau^{2-\Delta}}{2\left(|w-z|^{2}+\rho^{-2} \mp i \varepsilon\right)^{\Delta+2}}\left(\begin{array}{cccc}
0 & 0 & 0 & 0 \\
0 & \frac{4(\bar{w}-\bar{z})^{2}}{\rho^{\Delta+4}} & \frac{2(\bar{w}-\bar{z})^{3}}{\rho^{\Delta+1}} & \frac{-2(\bar{w}-\bar{z})}{\rho^{\Delta+3}} \\
0 & \frac{2(\bar{w}-\bar{z})^{3}}{\rho^{\Delta+1}} & \frac{(\bar{w}-\bar{z})^{4}}{\rho^{\Delta-2}} & \frac{-(\bar{w}-\bar{z})^{2}}{\rho^{\Delta}} \\
0 & \frac{-2(\bar{w}-\bar{z})}{\rho^{\Delta+3}} & \frac{-(\bar{w}-\bar{z})^{2}}{\rho^{\Delta}} & \frac{1}{\rho^{\Delta+2}}
\end{array}\right) .
$$

For $\Delta=2$ one finds the simple result $[37,39]$

$$
h_{\mu \nu ; w w}^{2}=\frac{1}{\tau^{2}} h_{\mu \nu ; w w}^{\text {Goldstone }},
$$

\footnotetext{
${ }^{7}$ In [37] this mode is denoted $\widetilde{h^{\Delta=0}} \mu \nu ; w w$, where the tilde indicates the fact that it is the shadow of a mode with conformal weight 0 in the basis (8.2).

${ }^{8}$ We note that these modes generically have radiation flux though $\mathcal{C S}^{+}[37]$ and so do not obey the boundary conditions for the charge defined on that surface.
} 
which is not a pure diffeomorphism. The symplectic product (4.8) of these two modes on $H_{3}^{+}$is ${ }^{9}$

$$
\left(h_{w w}^{\text {Goldstone }}, h_{v v}^{2+i \varepsilon}\right)_{H_{3}^{+}}=\frac{\pi}{48(w-v)^{4}} \delta(\varepsilon) .
$$

This resembles an off-diagonal central charge. The symplectic product over a complete spacelike Cauchy slice $\Sigma_{3}$ is

$$
\left(h_{w w}^{\text {Goldstone }}, h_{v v}^{2+i \varepsilon}\right)_{\Sigma_{3}}=-\varepsilon \frac{i \pi^{2}}{6(w-v)^{4}} \delta(\varepsilon)
$$

Naïvely, the right hand side vanishes due to the factor of the imaginary part of the conformal weight $\varepsilon$. However, we leave it in this form as in some contexts there may be compensating conformally soft poles in $\varepsilon$. A second conformal weight $(2,0)$ operator on the celestial sphere (in addition to $\mathcal{T}_{w w}^{K M R S}$ ) can be constructed explicitly from the mode (8.4). Potential implications of two weight $(2,0)$ operators for the structure of the soft gravitational $\mathcal{S}$ matrix are left to future work.

\section{Acknowledgments}

This work was supported in part by NSF grants 1205550, 1745303, and 1144152, the John Templeton Foundation, and the Hertz Foundation. We are grateful to Laura Donnay, Dan Kapec, Monica Pate, Andrea Puhm, Ana-Maria Raclariu, and Shu-Heng Shao for useful discussions.

\section{A Linearized Einstein equations}

In radial gauge, $h_{\tau \mu}=0$, the Einstein equations take the form

$$
\begin{aligned}
G_{\tau \tau}= & \frac{\rho^{2}}{\tau^{4}}\left(\tau \partial_{\tau}+\rho \partial_{\rho}+3-\frac{2}{\rho^{2}} \partial_{z} \partial_{\bar{z}}\right) h_{\rho \rho}+\frac{2}{\rho \tau^{4}}\left(\rho \partial_{\rho}+2\right)\left(\partial_{\bar{z}} h_{\rho z}+\partial_{z} h_{\rho \bar{z}}\right) \\
& +\frac{2}{\rho^{4} \tau^{4}}\left(\partial_{\bar{z}}^{2} h_{z z}+\partial_{z}^{2} h_{\bar{z} \bar{z}}\right)+\frac{2}{\rho^{2} \tau^{4}}\left(2 \tau \partial_{\tau}-\rho^{2} \partial_{\rho}^{2}-\frac{2}{\rho^{2}} \partial_{z} \partial_{\bar{z}}-2\right) h_{z \bar{z}} \\
G_{\tau \rho}= & \left(\tau \partial_{\tau}-2\right)\left[\frac{\rho}{\tau^{3}} h_{\rho \rho}+\frac{1}{\rho^{2} \tau^{3}}\left(\partial_{\bar{z}} h_{\rho z}+\partial_{z} h_{\rho \bar{z}}\right)+\frac{2}{\rho^{3} \tau^{3}}\left(1-\rho \partial_{\rho}\right) h_{z \bar{z}}\right] \\
G_{\rho \rho}= & -\frac{1}{\tau^{2}} h_{\rho \rho}-\frac{2}{\rho^{3} \tau^{2}}\left(\partial_{\bar{z}} h_{\rho z}+\partial_{z} h_{\rho \bar{z}}\right)-\frac{2}{\rho^{6} \tau^{2}}\left(\partial_{\bar{z}}^{2} h_{z z}+\partial_{z}^{2} h_{\bar{z} \bar{z}}\right) \\
& -\frac{2}{\rho^{4} \tau^{2}}\left(\tau^{2} \partial_{\tau}^{2}-\tau \partial_{\tau}-\rho \partial_{\rho}+2-\frac{2}{\rho^{2}} \partial_{z} \partial_{\bar{z}}\right) h_{z \bar{z}} \\
G_{\tau z}= & \left(\tau \partial_{\tau}-2\right)\left[-\frac{\rho^{2}}{2 \tau^{3}} \partial_{z} h_{\rho \rho}+\frac{\rho}{2 \tau^{3}}\left(\rho \partial_{\rho}+3\right) h_{\rho z}+\frac{1}{\rho^{2} \tau^{3}}\left(\partial_{\bar{z}} h_{z z}-\partial_{z} h_{z \bar{z}}\right)\right] \\
G_{\rho z}= & \frac{\rho}{2 \tau^{2}} \partial_{z} h_{\rho \rho}+\frac{1}{2 \tau^{2}}\left(\tau^{2} \partial_{\tau}^{2}-\tau \partial_{\tau}-\frac{2}{\rho^{2}} \partial_{z} \partial_{\bar{z}}\right) h_{\rho z}+\frac{1}{\rho^{2} \tau^{2}} \partial_{z}^{2} h_{\rho \bar{z}} \\
& +\frac{1}{\rho^{3} \tau^{2}}\left(\rho \partial_{\rho}-2\right)\left(\partial_{\bar{z}} h_{z z}-\partial_{z} h_{z \bar{z}}\right)
\end{aligned}
$$

\footnotetext{
${ }^{9}$ Useful formulae for evaluating these integrals can be found in $[39,44,45]$.
} 


$$
\begin{aligned}
G_{z z}= & -\frac{\rho^{2}}{2 \tau^{2}} \partial_{z}^{2} h_{\rho \rho}+\frac{\rho}{\tau^{2}}\left(\rho \partial_{\rho}+1\right) \partial_{z} h_{\rho z}+\frac{1}{2 \tau^{2}}\left(\tau^{2} \partial_{\tau}^{2}-\tau \partial_{\tau}-\rho^{2} \partial_{\rho}^{2}+\rho \partial_{\rho}\right) h_{z z} \\
G_{z \bar{z}}= & \frac{\rho^{4}}{4 \tau^{2}}\left(-\tau^{2} \partial_{\tau}^{2}+\tau \partial_{\tau}-\rho \partial_{\rho}-4+\frac{2}{\rho^{2}} \partial_{z} \partial_{\bar{z}}\right) h_{\rho \rho}+\frac{1}{2 \tau^{2}}\left(-\tau^{2} \partial_{\tau}^{2}+\tau \partial_{\tau}+\rho^{2} \partial_{\rho}^{2}-\rho \partial_{\rho}\right) h_{z \bar{z}} \\
& -\frac{\rho}{2 \tau^{2}}\left(\rho \partial_{\rho}+1\right)\left(\partial_{\bar{z}} h_{\rho z}+\partial_{z} h_{\rho \bar{z}}\right) .
\end{aligned}
$$

Note that the Einstein equations completely decouple under different $\tau$ scalings, so it is natural to decompose the metric in a $\tau$ expansion as $h_{\mu \nu}=\sum_{n} \tau^{-n} h_{\mu \nu}^{(n)}(\rho, z, \bar{z})$.

Working in "on-shell gauge" of the free Einstein equations we arrived at an equation for $h_{z z}^{(0)}$ by itself. The gauge assumes that

$$
\begin{aligned}
X^{\mu} h_{\mu \nu} & =0 \\
\nabla^{\mu} h_{\mu \nu} & =0 \\
g^{\mu \nu} h_{\mu \nu} & =0,
\end{aligned}
$$

where $X^{\mu}$ are Cartesian coordinates. Note that in hyperbolic coordinates (A.2) is equivalent to $h_{\tau \mu}=0$. The $G_{\tau \mu}$ equations all follow from these gauge conditions, and $G_{\rho \rho}$ and $G_{z \bar{z}}$ are equivalent in this gauge. The $G_{z z}$ equation can be used to eliminate $h_{\rho z}^{(0)}$ in favor of $h_{z z}^{(0)}$ (up to integration constants). Plugging into $G_{\rho z}$ gives

$0=\rho^{4}\left(\rho \partial_{\rho}+4\right)\left(\rho \partial_{\rho}+2\right)\left(\rho \partial_{\rho}-2\right) \rho \partial_{\rho} h_{z z}^{(0)}+8 \rho^{2}\left(\rho \partial_{\rho}+2\right)\left(\rho \partial_{\rho}-2\right) \partial_{z} \partial_{\bar{z}} h_{z z}^{(0)}+16\left(\partial_{z} \partial_{\bar{z}}\right)^{2} h_{z z}^{(0)}$.

Given a solution of (A.5), the other metric components in the gauge (A.2) are constrained.

Linearized metric perturbations along a vector field $\xi^{\mu} \partial_{\mu}$ are given by

$$
\begin{aligned}
\delta g_{\tau \tau} & =\frac{2}{\tau} \tau \partial_{\tau} \xi_{\tau} \\
\delta g_{\tau \rho} & =\partial_{\rho} \xi_{\tau}+\frac{1}{\tau}\left(\tau \partial_{\tau}-2\right) \xi_{\rho} \\
\delta g_{\rho \rho} & =2\left(\partial_{\rho}+\frac{1}{\rho}\right) \xi_{\rho}-\frac{2 \tau}{\rho^{2}} \xi_{\tau} \\
\delta g_{\tau z} & =\frac{1}{\tau}\left(\tau \partial_{\tau}-2\right) \xi_{z}+\partial_{z} \xi_{\tau} \\
\delta g_{\rho z} & =\left(\partial_{\rho}-\frac{2}{\rho}\right) \xi_{z}+\partial_{z} \xi_{\rho} \\
\delta g_{z z} & =2 \partial_{z} \xi_{z} \\
\delta g_{z \bar{z}} & =\partial_{z} \xi_{\bar{z}}+\partial_{\bar{z}} \xi_{z}+\rho^{3} \xi_{\rho}-\rho^{2} \tau \xi_{\tau} .
\end{aligned}
$$

Setting $\delta g_{\tau \mu}=0$, we must have $\partial_{\tau} \xi_{\tau}=0$. We can satisfy the conditions with $\xi_{\tau}=0$ and $\xi_{\rho}, \xi_{z}, \xi_{\bar{z}} \propto \tau^{2}$, but this is not completely general. We can also let $\xi_{\tau}(\rho, z, \bar{z})$ be a generic function and choose the $\mathcal{O}(\tau)$ pieces of the other components accordingly. The general solution, using $\tau$ weight notation $\xi_{\mu}^{(n)}$, is

$$
\begin{aligned}
& \xi_{\tau}=\xi_{\tau}^{(0)}(\rho, z, \bar{z}) \\
& \xi_{\rho}=\tau^{2} \xi_{\rho}^{(-2)}(\rho, z, \bar{z})+\tau \partial_{\rho} \xi_{\tau}^{(0)} \\
& \xi_{z}=\tau^{2} \xi_{z}^{(-2)}(\rho, z, \bar{z})+\tau \partial_{z} \xi_{\tau}^{(0)} \\
& \xi_{\bar{z}}=\tau^{2} \xi_{\bar{z}}^{(-2)}(\rho, z, \bar{z})+\tau \partial_{\bar{z}} \xi_{\tau}^{(0)} .
\end{aligned}
$$


Here we treat the $\tau$ dependence as not included in $\xi_{\mu}^{(n)}$. We see the free data for these residual diffeomorphisms are four free functions of three variables, and that these free functions only affect the $h_{\mu \nu}^{(-1)}$ and $h_{\mu \nu}^{(-2)}$ pieces of the metric in hyperbolic coordinates.

Open Access. This article is distributed under the terms of the Creative Commons Attribution License (CC-BY 4.0), which permits any use, distribution and reproduction in any medium, provided the original author(s) and source are credited.

\section{References}

[1] J. de Boer and S.N. Solodukhin, A holographic reduction of Minkowski space-time, Nucl. Phys. B 665 (2003) 545 [hep-th/0303006] [INSPIRE].

[2] M. Campiglia and A. Laddha, Asymptotic symmetries of QED and Weinberg's soft photon theorem, JHEP 07 (2015) 115 [arXiv: 1505. 05346] [INSPIRE].

[3] M. Campiglia and A. Laddha, Asymptotic symmetries of gravity and soft theorems for massive particles, JHEP 12 (2015) 094 [arXiv: 1509. 01406] [INSPIRE].

[4] C. Cheung, A. de la Fuente and R. Sundrum, $4 D$ scattering amplitudes and asymptotic symmetries from 2D CFT, JHEP 01 (2017) 112 [arXiv:1609.00732] [INSPIRE].

[5] M. Gary, S.B. Giddings and J. Penedones, Local bulk S-matrix elements and CFT singularities, Phys. Rev. D 80 (2009) 085005 [arXiv:0903.4437] [INSPIRE].

[6] A.L. Fitzpatrick and J. Kaplan, Scattering States in AdS/CFT, arXiv:1104.2597 [INSPIRE].

[7] G. Barnich and C. Troessaert, Aspects of the BMS/CFT correspondence, JHEP 05 (2010) 062 [arXiv: 1001.1541] [INSPIRE].

[8] G. Barnich and C. Troessaert, Supertranslations call for superrotations, PoS (CNCFG2010) 010 (2010) [Ann. U. Craiova Phys. 21 (2011) S11] [arXiv:1102.4632] [INSPIRE].

[9] G. Barnich and C. Troessaert, BMS charge algebra, JHEP 12 (2011) 105 [arXiv:1106. 0213] [INSPIRE].

[10] G. Barnich and C. Troessaert, Finite BMS transformations, JHEP 03 (2016) 167 [arXiv: 1601.04090] [INSPIRE].

[11] H. Bondi, Gravitational Waves in General Relativity, Nature 186 (1960) 535 [INSPIRE].

[12] H. Bondi, M.G.J. van der Burg and A.W.K. Metzner, Gravitational waves in general relativity. 7. Waves from axisymmetric isolated systems," Proc. Roy. Soc. Lond. A 269 (1962) 21.

[13] R. Sachs, Asymptotic symmetries in gravitational theory, Phys. Rev. 128 (1962) 2851 [INSPIRE].

[14] D. Kapec, V. Lysov, S. Pasterski and A. Strominger, Semiclassical Virasoro symmetry of the quantum gravity $\mathcal{S}$-matrix, JHEP 08 (2014) 058 [arXiv: 1406.3312] [INSPIRE].

[15] D. Kapec, P. Mitra, A.-M. Raclariu and A. Strominger, 2D Stress Tensor for $4 D$ Gravity, Phys. Rev. Lett. 119 (2017) 121601 [arXiv:1609.00282] [INSPIRE].

[16] F. Cachazo and A. Strominger, Evidence for a New Soft Graviton Theorem, arXiv: 1404.4091 [INSPIRE]. 
[17] M. Campiglia, Null to time-like infinity Green's functions for asymptotic symmetries in Minkowski spacetime, JHEP 11 (2015) 160 [arXiv: 1509.01408] [INSPIRE].

[18] A. Ashtekar and M. Streubel, Symplectic Geometry of Radiative Modes and Conserved Quantities at Null Infinity," Proc. Roy. Soc. Lond. A 376 (1981) 585.

[19] T. Dray and M. Streubel, Angular momentum at null infinity, Class. Quant. Grav. 1 (1984) 15 [INSPIRE].

[20] A. Strominger, On BMS Invariance of Gravitational Scattering, JHEP 07 (2014) 152 [arXiv: 1312.2229] [INSPIRE].

[21] V. Balasubramanian and P. Kraus, A stress tensor for Anti-de Sitter gravity, Commun. Math. Phys. 208 (1999) 413 [hep-th/9902121] [INSPIRE].

[22] J.D. Brown and M. Henneaux, Central Charges in the Canonical Realization of Asymptotic Symmetries: An example from Three-Dimensional Gravity, Commun. Math. Phys. 104 (1986) 207 [INSPIRE].

[23] J.M. Maldacena, The large $N$ limit of superconformal field theories and supergravity, Int. J. Theor. Phys. 38 (1999) 1113 [hep-th/9711200] [INSPIRE].

[24] O. Aharony, S.S. Gubser, J.M. Maldacena, H. Ooguri and Y. Oz, Large N field theories, string theory and gravity, Phys. Rept. 323 (2000) 183 [hep-th/9905111] [INSPIRE].

[25] C. Fefferman and C.R. Graham, Conformal invariants, in Élie Cartan et les mathématiques d'aujourd'hui - Lyon, 25-29 juin 1984, no. S131 in Astérisque, pp. 95-116. Société mathématique de France, (1985), http://www.numdam.org/item/AST_1985_S131__95_0.

[26] C. Fefferman and C.R. Graham, The ambient metric, Ann. Math. Stud. 178 (2011) 1 [arXiv:0710.0919] [INSPIRE].

[27] G.J. Zuckerman, Action principles and global geometry, Conf. Proc. C 8607214 (1986) 259 [INSPIRE].

[28] C. Crnkovic and E. Witten, Covariant description of canonical formalism in geometrical theories, in S.W. Hawking and W. Israel eds., Three hundred years of gravitation, (1987), pp. 676-684, [INSPIRE].

[29] J. Lee and R.M. Wald, Local symmetries and constraints, J. Math. Phys. 31 (1990) 725 [INSPIRE].

[30] V. Iyer and R.M. Wald, Some properties of Noether charge and a proposal for dynamical black hole entropy, Phys. Rev. D 50 (1994) 846 [gr-qc/9403028] [INSPIRE].

[31] V. Iyer and R.M. Wald, A comparison of Noether charge and Euclidean methods for computing the entropy of stationary black holes, Phys. Rev. D 52 (1995) 4430 [gr-qc/9503052] [INSPIRE].

[32] R.M. Wald and A. Zoupas, A general definition of 'conserved quantities' in general relativity and other theories of gravity, Phys. Rev. D 61 (2000) 084027 [gr-qc/9911095] [INSPIRE].

[33] G. Barnich and F. Brandt, Covariant theory of asymptotic symmetries, conservation laws and central charges, Nucl. Phys. B 633 (2002) 3 [hep-th/0111246] [INSPIRE].

[34] S.G. Avery and B.U.W. Schwab, Noether's second theorem and Ward identities for gauge symmetries, JHEP 02 (2016) 031 [arXiv:1510.07038] [INSPIRE].

[35] T. He, V. Lysov, P. Mitra and A. Strominger, BMS supertranslations and Weinberg's soft graviton theorem, JHEP 05 (2015) 151 [arXiv:1401.7026] [INSPIRE]. 
[36] S. Pasterski, S.-H. Shao and A. Strominger, Flat Space Amplitudes and Conformal Symmetry of the Celestial Sphere, Phys. Rev. D 96 (2017) 065026 [arXiv:1701.00049] [InSPIRE].

[37] L. Donnay, A. Puhm and A. Strominger, Conformally Soft Photons and Gravitons, JHEP 01 (2019) 184 [arXiv: 1810.05219] [InSPIRE].

[38] S. Pasterski, A. Strominger and A. Zhiboedov, New Gravitational Memories, JHEP 12 (2016) 053 [arXiv : 1502.06120] [INSPIRE].

[39] S. Pasterski and S.-H. Shao, Conformal basis for flat space amplitudes, Phys. Rev. D 96 (2017) 065022 [arXiv:1705.01027] [INSPIRE].

[40] D. Christodoulou and S. Klainerman, The Global nonlinear stability of the Minkowski space, Princeton University Press (1993).

[41] D. Kapec, M. Pate and A. Strominger, New Symmetries of QED, Adv. Theor. Math. Phys. 21 (2017) 1769 [arXiv:1506.02906] [inSPIRE].

[42] A. Nande, M. Pate and A. Strominger, Soft Factorization in QED from 2D Kac-Moody Symmetry, JHEP 02 (2018) 079 [arXiv: 1705. 00608] [INSPIRE].

[43] A. Strominger, Magnetic Corrections to the Soft Photon Theorem, Phys. Rev. Lett. 116 (2016) 031602 [arXiv:1509.00543] [INSPIRE].

[44] F.A. Dolan and H. Osborn, Conformal partial waves and the operator product expansion, Nucl. Phys. B 678 (2004) 491 [hep-th/0309180] [InSPIRE].

[45] D. Simmons-Duffin, Projectors, Shadows and Conformal Blocks, JHEP 04 (2014) 146 [arXiv: 1204.3894] [INSPIRE]. 